\title{
Paraneoplastic pemphigus with clinical features of lichen planus associated with low-grade B cell lymphoma
}

\author{
Sónia Coelho, MD, José Pedro Reis, MD, Oscar Tellechea, MD, PhD, Américo Figueiredo, MD, \\ $\mathrm{PhD}$, and Martin Black, $\mathrm{MD}, \mathrm{PhD}$
}

From the Department of Dermatology, University Hospital, Coimbra, Portugal, St John's Institute of Dermatology, St Thomas' Hospital, London, UK

\author{
Correspondence \\ Sónia Coelho \\ Clínica de Dermatologia, Hospital da \\ Universidade \\ P.3000-075 Coimbra \\ Portugal \\ E-mail: sonia.alexcoelho@clix.pt
}

\begin{abstract}
Background Neoplasia-induced lichen planus is described as a cell-mediated reaction to unknown epithelial antigens. Paraneoplastic pemphigus (PNP), characterized by the presence of a specific array of autoantibodies, probably represents a different form of presentation of the same autoimmune syndrome where the mucocutaneous expression depends on the dominant pathologic mechanism.

Methods The authors report a case of PNP with predominant lichen planus-like lesions and review the relevant literature. We observed a 74-year-old female with vesico-bullous, erosive, target-shaped and flat papular lichenoid lesions on the lower legs, palms and soles, evolving for 3 weeks. Histopathology revealed a lichenoid dermatitis. Direct immunofluorescence showed C3 deposition around keratinocytes and epidermal IgG intranuclear deposition. Indirect immunofluorescence revealed circulating lgG with intercellular staining on rat bladder substrate. Immunoblotting demonstrated bands of 130, 190, 210 and $250 \mathrm{kDa}$ antigens. A pararenal B cell lymphoma was found.

Results Oral corticotherapy with $40 \mathrm{mg}$ prednisolone daily was initiated with a good cutaneous response. Four months later, cyclophosphamide ( $50 \mathrm{mg} /$ day) was introduced because of a discrete enlargement of the pararenal mass. The patient died on the seventh month of follow up as a result of respiratory insufficiency.

Conclusion PNP has different forms of presentation and the lack of a consensus about diagnostic criteria may contribute to underdiagnosed cases. Advances on the knowledge of the sensitivity and specificity of diagnostic criteria have allowed a better accuracy of diagnosis.
\end{abstract}

\section{Introduction}

Paraneoplastic pemphigus (PNP) is an autoimmune bullous disease with mucocutaneous involvement, individualized in I990 by Anhalt et al. ${ }^{\text {I }}$

Before this nosological individualization, several cases were reported in the literature as unusual pemphigus vulgaris, unusual erythema multiforme or as unusual paraneoplastic bullous diseases. ${ }^{2,3}$

Since the first description, different forms of presentation of PNP have been recognized and advances on the sensitivity and specificity of the diagnostic criteria originally proposed have been made.

We report a case of PNP with predominant lichen planuslike lesions and review the relevant literature.

\section{Case Report}

We observed a 74-year-old White woman with mucocutane- with painful, erythemato-papular, vesico-bullous, erosive and target-shaped lesions on the lower legs (Fig. I), palms, soles and abdomen along with flat-topped violaceous lichenoid papules in the same distribution. Erosive and lichenoid lesions of oral mucosa, lips and eyelids were also present. Lichenoid lesions predominated and were found isolated, or overlapping the other ones. She had mild conjunctivitis, no genital involvement, and the physical examination was otherwise normal. Her medical history was noncontributory. The clinical impression was of lichen planus pemphigoid.

Histopathology of lichenoid and target lesions revealed an interface cell-rich dermatitis, accompanied by dermalepidermal hydropic changes (Fig. 2). No acantholysis was found. Direct immunofluorescence (DIF) on perilesional skin displayed colloidal body deposition at the Dermoepidermal junction (DEJ) and papillary dermis with $\operatorname{IgG}$ and $\mathrm{C}_{3}, \mathrm{C}_{3}$ focal deposition in vessel walls of papillary dermis and around basal keratinocytes, and focal epidermal IgG intranuclear deposition. Indirect immunofluorescence (IIF) identified circulating IgG with cell surface staining present to a titer of I : Io on monkey 


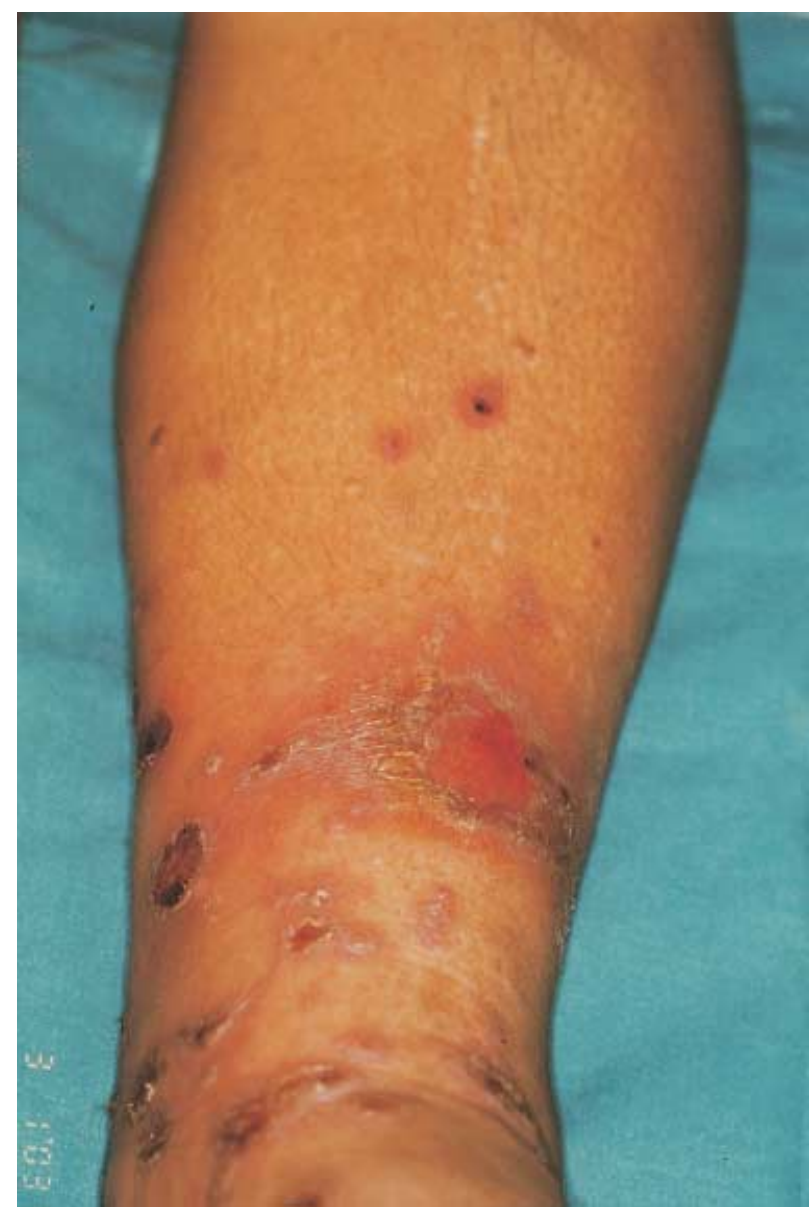

Figure 1 Vesicles, erosions and target-shaped lesions on the lower leg. Some flat topped violaceous lichenoid papules are also observed

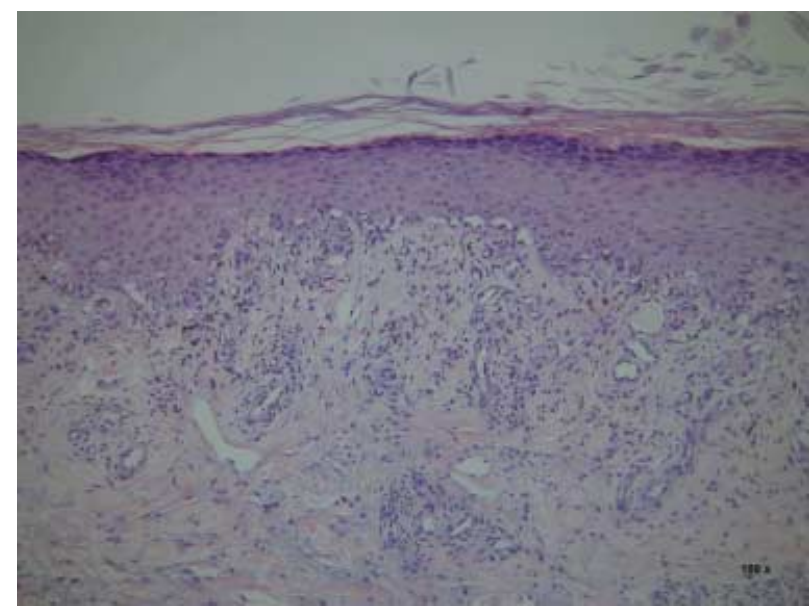

Figure 2 Histopathology in target lesion revealed an interface cell-rich dermatitis without acantholysis, accompanied by dermal-epidermal hydropic changes (stain type - Hematoxylin and eosin; original magnification $\times$ IOO)

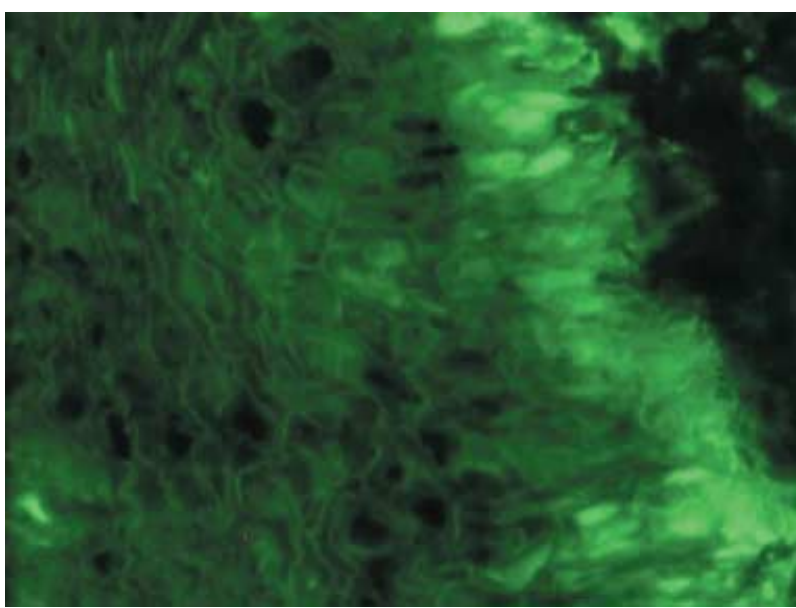

Figure 3 Indirect immunofluorescence on rat bladder showing intercellular staining with IgG (stain type - Fluorescein; original magnification $\times 400$ )

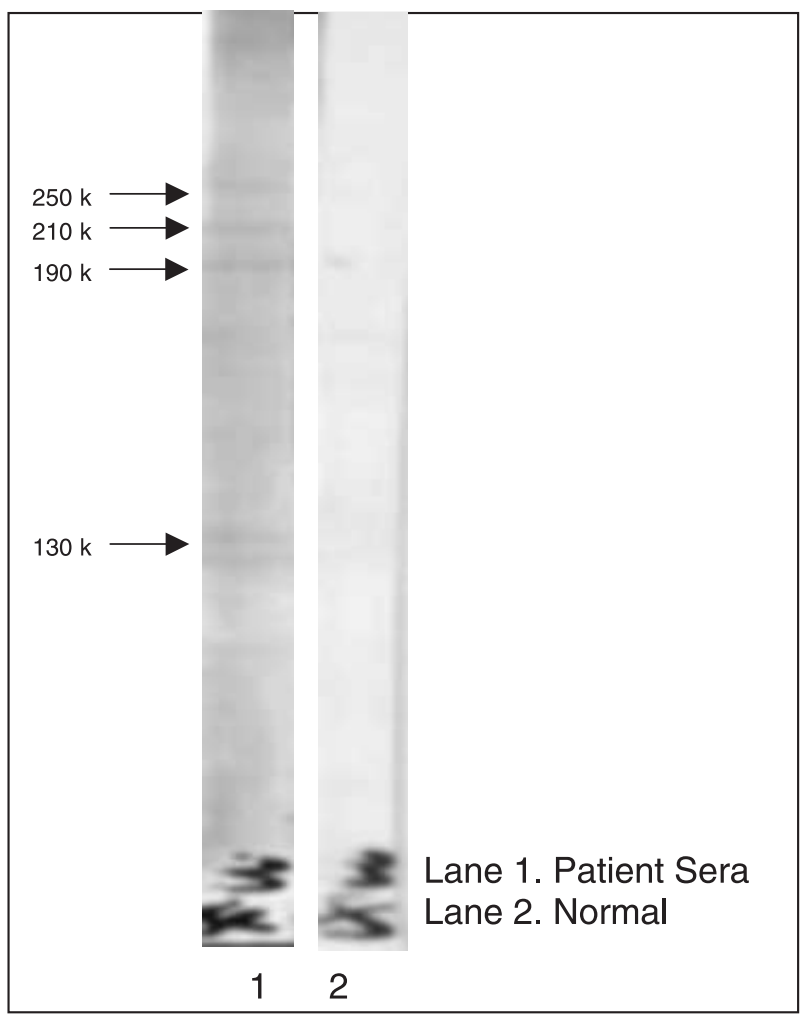

Figure 4 Immunoprecipitation preparation demonstrating the characteristic pattern of PNP

esophagus and rat bladder (Fig. 3) along with nuclear epithelial staining with IgG on normal skin and monkey esophagus. The patient's sera immunoprecipitated an antigenic complex composed of several proteins - I30 kDa, I90 kDa, 2 Io kDa and $250 \mathrm{kDa}$ - that, respectively, correspond to desmoglein 3, periplakin, envoplakin and desmoplakin I (Fig. 4). All these findings were consistent with paraneoplastic pemphigus. 
A left pararenal mass of $6.5 \times 4 \mathrm{~cm}$ was identified on CT scan. Pathological examination of the mass revealed a lowgrade $\mathrm{B}$ cell lymphoma which was bcl 2 positive, $\mathrm{CD}_{2} \mathrm{O}$ and $\mathrm{CD}$ Io negative. The relevant abnormal laboratory findings included a positive serum Antinuclear Antibodies (ANA) with homogeneous pattern and an IgM lambda monoclonal gammopathy. Oral corticotherapy with $40 \mathrm{mg}$ prednisolone daily was initiated along with topical application of betamethasone. The lymphoma, at this time, was maintained on surveillance and no specific treatment was initiated.

Almost complete clearing of lesions was observed by the third month and tapering of prednisolone was therefore undertaken. This did not modify the dermatological picture. By the fourth month of follow up, warfarin $(5 \mathrm{mg} /$ day $)$ and cyclophosphamide (50 mg/day) were initiated, respectively, as a result of a leg deep vein thrombosis and a discrete enlargement of the pararenal mass. Sparse lichenoid lesions were the only cutaneous manifestation.

Two months latter she was hospitalized in another institution because of dyspnea and fatigue, dying from respiratory failure after a few weeks. The arterial blood gas analysis and chest radiography were inconsistent with the severity of the dyspnea. The probable cause for this fatal pulmonary complication was the development of obliterative bronchiolitis, as reported in other similar cases. Unfortunately a biopsy for the diagnosis of obliterant bronchiolitis was not undertaken.

\section{Discussion}

The mucocutaneous lesions of PNP have several clinical presentations. ${ }^{\mathrm{I}, 2,4}$ Patients can present a pruritic skin eruption that is bullous pemphigoid-like, with vesicles, bullae, erosions and crusting, but can also have erythematous papules with central vesiculation resembling erythema multiforme. The trunk, proximal extremities, head and neck are the most commonly affected regions, although in our patient they predominated on the lower legs. Nikolsky sign can occasionally be elicited. ${ }^{5}$ PNP spectrum also includes, a lichen planus pemphigoid-like eruption that can precede or follow the symptoms of the neoplasm..$^{5-\mathrm{ro}}$ Blisters and lichenoid lesions on the palms and soles are frequently seen, helping to differentiate it from pemphigus vulgaris. An intractable stomatitis, similar to that of pemphigus vulgaris, often confused with herpetic infection, ${ }^{4}$ is usually the inaugural manifestation, with cutaneous lesions developing shortly after. ${ }^{\mathrm{I}}$ Pseudomembranous conjunctivitis evolving to scaring is also frequent. ${ }^{2,4}$

Lesions may also be found on laryngeal, nasopharyngeal, esophageal, lingual, buccal, gingival, labial, gastrointestinal, vaginal and penile mucosa. ${ }^{2}$

Respiratory involvement with dyspnea resulting from obliterant bronchiolitis is a frequent cause of death. This is the consequence of intraluminal shedding of mucosal epithelium, involved in the immune attack as a result of its richness in desmoplakins. ${ }^{2,4}$ Patients usually have arterial blood gas analysis and chest radiography inconsistent with the severity of the dyspnea.

The disease has a broad geographic distribution, with similar gender predominance and mean age of onset at around 5 I years. ${ }^{2}$ Of the several neoplasms associated with PNP, $86 \%$ are lymphoproliferative disorders. The most commonly found in decreasing order of frequency are ${ }^{2}$ :

I non-Hodgkin's lymphoma;

2 chronic lymphocytic leukemia;

3 Castleman's tumor;

4 thymoma (malignant and benign);

5 poorly-differentiated sarcoma;

6 Waldenström's macroglobulinemia;

7 inflammatory fibrosarcoma;

8 bronchogenic squamous cell carcinoma;

9 round-cell liposarcoma;

Io Hodgkin's disease; and

II $\mathrm{T}$ cell lymphoma.

In one third of cases, the neoplasm is found after the development of mucocutaneous disease. ${ }^{2,3}$

On histological examination, suprabasilar acantholysis, dermoepidermal cleavage, keratinocyte necrosis, epidermal necrosis, interface changes with dense lichenoid infiltrate in the papillary dermis and eosinophilic spongiosis, can be found. ${ }^{\text {II }}$

Direct immunofluorescence (DIF) on perilesional skin should reveal $\operatorname{IgG}$ and/or $\mathrm{C}_{3}$ cell surface deposition, like in pemphigus vulgaris, sometimes with sparse focal deposition. Granularlinear deposits of $\mathrm{C}_{3}$ at the basement membrane zone may also be seen. ${ }^{2,11}$ The possibility of false-negative results demands, if necessary, repeated exams. ${ }^{2}$

The IIF pattern is identical to that of pemphigus vulgaris and pemphigus foliaceous, with staining of the epidermal cell surface and/or basement membrane zone., ${ }^{2, \mathrm{II}, \mathrm{I2}}$ Rat bladder is the preferred substrate, because of its richness in desmosomes, having a sensitivity of $86 \%$ and a specificity of $98 \%$ in the screening of PNP. ${ }^{2,11,13,14}$ These antibodies can bind other substrates with lower sensitivity and specificity. ${ }^{2,13}$ This may explain why the PNP spectrum goes beyond the mucocutaneous manifestations and includes antibody deposition on different organs namely respiratory epithelium, colon epithelium, intercalated disks of myocardium, skeletal muscle and thyroid epithelium. ${ }^{2}$

Because of its specificity, IIF on rat bladder can be used instead of immunoprecipitation studies. ${ }^{\text {II }}$

PNP sera can recognize one or more of several proteins (Table I). ${ }^{2,15}$ It is probable that desmoglein 3 , because of its transmembrane location, will be the target antigen in PNP, initiating the acantholytic process. Antibodies to desmoglein 3 have been proven to be pathogenic and it has been recently demonstrated that they can be detected by ELISA in I00\% of the patients. ${ }^{\mathrm{II}, \mathrm{I}}$ This first attack would expose additional 
Table 1 PNP autoantigens

\begin{tabular}{lr}
\hline Desmoplakin I & $250 \mathrm{kDa}$ \\
Desmoplakin II & $215 \mathrm{kDa}$ \\
Bullous pemphigoid antigen I (BPAg I) & $230 \mathrm{kDa}$ \\
Envoplakin & $210 \mathrm{kDa}$ \\
Periplakin & $190 \mathrm{kDa}$ \\
Desmoglein 1 & $165 \mathrm{kDa}$ \\
Desmoglein 3 & $130 \mathrm{kDa}$ \\
Undetermined trans-membranous antigen & $170 \mathrm{kDa}$ \\
\hline
\end{tabular}

Table 2 Anhalt et al. criteria

\section{Clinical}

Painful mucosal ulcerations and blisters and a polymorphous skin eruption, with papular lesions progressing to blisters and erosive lesions on the trunk, extremities, palms and soles, in the context of occult neoplasm.

\section{Histologic findings}

Vacuolar interface change, keratinocytes necrosis and intra-epidermal acantholysis.

DIF

Deposition of IgG and C3 in the intercellular spaces, and granular-linear complement deposition along the epidermal basement membrane zone.

IIF

Serum antibodies that bind not only to cell surfaces of skin and mucosa in a pattern typical of pemphigus vulgaris, but also to simple, columnar, and transitional epithelia.

Immunoprecipitation

Serum antibodies that recognize epidermal antigens of 250, 230, 210, 190 kDa.

epitopes and will lead to the subsequent formation of antibodies to the intracellular plakins (epitope spreading theory). ${ }^{17}$ However, patients with pemphigus vulgaris do not have antibodies to the plakin family, supposing the participation of other mechanisms. ${ }^{2}$ Indeed, a pathogenic role for most of PNP-identified antibodies is still to be determined.

It should be emphasized that when PNP is suspected, it is of paramount importance to seek an occult neoplasm, especially with CT scan of the chest, abdomen and pelvis. ${ }^{2}$

Since $1990^{\mathrm{I}}$ (Table 2), several diagnostic criteria have been proposed, although still lacking a consensus about this issue.

In 1993 Camisa and Helm ${ }^{\mathrm{I} 8}$ proposed a modification of the Anhalt et al. criteria (Table 3). In a recent study, from Joly et al., ${ }^{\text {II }}$ patients with different types of pemphigus associated or not with neoplasia were reviewed in order to evaluate the sensitivity and specificity of the diagnostic criteria of PNP. The conclusion was that one clinical criteria and two biological criteria had an elevated sensitivity $(82-86 \%)$ and specificity $(83-100 \%)$ for PNP whatever the control group considered and a re-evaluation of previous diagnostic criteria was proposed (Table 4). The study also confirmed the accuracy of IIF on rat bladder and the high frequency of lymphoproliferative diseases associated with PNP.
Table 3 Camisa and Helm criteria

Major criteria

Polymorphous mucocutaneous eruption

Concurrent internal neoplasia

Characteristic serum immunoprecipitation findings

Minor criteria

Positive cytoplasmatic staining of rat bladder by IIF

Intercellular and basement membrane zone immunoreactants on direct immunofluorescence of perilesional tissue

Acantholysis in biopsy specimen from at least one anatomic site of involvement

Table 4 Joly et al. criteria

High specificity and sensitivity

1. Association with lymphoproliferative disorders

2. IIF positive on rat bladder

3. Immunoblotting detection of antibodies against envoplakin and periplakin

Low sensitivity and high specificity

1. Polymorphous eruption

2. Histology

3. DIF

4. Immunoblotting detection of antibodies against desmoplakins and BPAGI

Of the several etiopathogenic mechanisms proposed, the most consensual include:

I The tumor induces a cell-mediated lichenoid interface dermatitis that will uncover previously hidden antigens. ${ }^{9,17}$

2 An immune response against the tumor cross-reacting with normal epithelial proteins (antigenic mimicry). ${ }^{17}$

3 Dysregulation of cytokine production by tumor cells, with secretion of massive amounts of IL6. ${ }^{\text {I,2,17 }}$

All or some of these mechanisms can be present in a single patient, influencing the clinical, histological and immunological features.

Lichen planus is a mucocutaneous disease that can have several triggers, including neoplasms. The few well-characterized cases of LP in patients with malignancy are related to lichen planus pemphigoides. ${ }^{19}$

The lichen planus pemphigoides-like variant of PNP is characterized by tense blisters on previous lichenoid lesions or normal skin; lichenoid dermatitis on histological examination and eventual linear $\operatorname{IgG}$ and/or $\mathrm{C}_{3}$ deposition at the dermoepidermal junction on DIF. ${ }^{6}$ It lacks the circulating autoantibodies against 200 and $\mathrm{I} 80 \mathrm{kDa}$ epidermal antigens of LP pemphigoides ${ }^{20}$ and has the specific array of autoantibodies of PNP. However, in some patients with neoplasia and lichenoid dermatitis, auto-antibodies never occur. These cases have been designated neoplasia-induced lichen planus. ${ }^{\text {I9 }}$

We think that all these different forms of presentation are part of an auto-immune reaction mainly humoral in PNP and mainly cellular in neoplasia induced lichen planus, and 
evidence suggests that there are similar triggers. Why the different inaugural characteristics, sometimes with the same underlying neoplasm, is still obscure. It is also difficult to explain why in some patients, the clinical, histological and immunological features change over time with the appearance of acantholysis and autoantibodies, ${ }^{9}$ while in others this never happens. ${ }^{19}$ The term PNP should therefore be applied to patients that in spite of lichenoid lesions have the specific autoantibodies.

The prognosis of PNP associated with malignant neoplasms is poor with a rapidly fatal outcome in a few months. ${ }^{\mathrm{I}}{ }_{3}$ Camisa et al. report a case of prolonged survival suggesting a subgroup with a more favorable outcome. ${ }^{21}$ It seems that with indolent B-cell lymphomas, the paraneoplastic reaction could offer some protection against the progression of the disease. ${ }^{18}$ It should be kept in mind that disease progression doesn't parallel that of the neoplasm, as observed in our patient.

These neoplasms have been associated with other paraneoplastic syndromes, and some suggest the name "paraneoplastic autoimmune multiorgan syndrome" PAMS. ${ }^{22}$

A particularity of our clinical report was the absence of aggressive involvement of the oral mucosa. The patient also showed good response to oral corticotherapy, with a prolonged remission. The deep vein thrombosis was, in our opinion, another paraneoplastic manifestation and the presence of antinuclear antibodies an evidence of a patent dysimmunoreactivity. ${ }^{23}$

PNP manifested has lichenoid eruptions is more common then previously accepted and the potential role of cell-mediated immunity at the DEJ as a participant in the pathogenesis of PNP can't be underestimated.

\section{References}

I Anhalt GJ, Kim SC, Standley JR, et al. Paraneoplastic pemphigus. An autoimmune mucocutaneous disease associated with neoplasia. N Engl J Med I990; 323: I729-I735.

2 Robinson ND, Hashimoto T, Amagai M, et al. The new pemphigus variants. J Am Acad Dermatol I999 May; 40: 649-67I.

3 Anhalt GJ. Paraneoplastic pemphigus. Adv Dermatol I997; I2: 77-96.

4 Allen CM, Camisa C. Paraneoplastic pemphigus: a review of the literature. Oral Dis 2000; 6: 208-2I4.

5 Jansen T, Plewig G, Anhalt GJ. Paraneoplastic pemphigus with clinical features of erosive lichen planus associated with Castelman's tumor. Dermatology I995; I90: 245-250.

6 Krunic Al Kokai D, Bacetic B, et al. Retroperitoneal roundcell liposarcoma associated with paraneoplastic pemphigus presenting as lichen planus pemphigoides-like eruption. Int J Dermatol I997 July; 36: 526-529.
7 Hsiao CJ, Hsu MM, Lee JY, et al. Paraneoplastic pemphigus in association with retroperitoneal Castelman's disease presenting with lichen planus pemphigoides-like eruption. A case report and review of the literature. Br J Dermatol $200 \mathrm{I}$ February; I44: 372-376.

8 Stevens SR, Griffiths CE, Anhalt GJ, et al. Paraneoplastic pemphigus presenting as a lichen planus pemphigoides-like eruption. Arch Dermatol I993 July; I29: 866-869.

9 Bowen GM, Peters NT, Fivenson DP, et al. Lichenoid dermatitis in paraneoplastic pemphigus: a pathogenic trigger of epitope spreading? Arch Dermatol 2000 May; I36: 652656.

Io Beylot-Barry M, Doutre MS, Vergier B, et al. Lichenoid paraneoplastic pemphigus in low-grade nodular lymphoma. Ann Dermatol Venereol I998 October; I25: 720-723.

I I Joly P, Richard C, Gilbert D, et al. Sensitivity and specificity of clinical, histologic and immunologic features in the diagnosis of paraneoplastic pemphigus. J Am Acad Dermatol 2000 October; 43: 619-626.

I 2 Joly $\mathrm{P}$, Thomine E, Gilbert D, et al. Overlapping distribution of autoantibody specificities between pemphigus vulgaris and paraneoplastic pemphigus. J Invest Dermatol I994; I03: 65-72.

I3 Liu AY, Valenzuela R, Helm TN, et al. Indirect immunofluorescence on rat bladder transitional epithelium: a test with high specificity for paraneoplastic pemphigus. $J$ Am Acad Dermatol I993; 28: 696-699.

I4 Helou J, Allbritton J, Anhalt GJ. Accuracy of indirect immunofluorescence testing in the diagnosis of paraneoplastic pemphigus. J Am Acad Dermatol I995; 32: $44 \mathrm{I}-447$.

I 5 Hashimoto T, Amagai M, Watanabe K, et al. Characterization of paraneoplastic pemphigus autoantigens by immunoblot analysis. J Invest Dermatol I995; I04: 829-834.

I6 Amagai M, Nishikawa T, Noussari HC, et al. Antibodies against desmoglein 3 (pemphigus vulgaris antigen) are present in sera from patients with paraneoplastic pemphigus and cause acantholysis in vivo in neonatal mice. J Clin Invest I998; I02: 775-782.

I7 Chan LS. Epitope spreading in paraneoplastic pemphigus: autoimmune induction in antibody-mediated blistering skin diseases. Arch Dermatol 2000 May; 136: 663-664.

I 8 Camisa C, Helm TN. Paraneoplastic pemphigus is a distinct neoplasia-induced autoimmune disease. Arch Dermatol I993 July; I 29: 883-885.

I9 Helm TN, Camisa C, Liu AY, et al. Lichen planus associated with neoplasia: a cell-mediated immune response to tumor antigens? J Am Acad Dermatol 1994 February; 30: 219224.

20 Yoon KH, Kim SC, Kang DS, et al. Lichen planus pemphigoides with circulating autoantibodies against 200 and I $80 \mathrm{kDa}$ epidermal antigens. Eur J Dermatol 2000 April-May; Io: 2I 2-2 I4.

2 I Camisa C, Helm TN, Liu YC, et al. Paraneoplastic pemphigus: a report of three cases including one long term survivor. J Am Acad Dermatol 192 October; 27: 547-553. 
22 Nguyen VT, Ndoye A, Bassler KD, et al. Classification, clinical manifestations and immunological mechanisms of the epithelial variant of paraneoplastic autoimmune multiorgan syndrome: a reappraisal of paraneoplastic pemphigus. Arch Dermatol 200I February; I37: I93-206.
23 Mahler V, Antoni C, Anhalt GJ, et al. Graft versus host-like mucocutaneous eruptions with serological features of paraneoplastic pemphigus and systemic lupus erythematosus in a patient with non-Hodgkin's lymphoma. Dermatology I998 July; I97: 78-83. 
This document is a scanned copy of a printed document. No warranty is given about the accuracy of the copy. Users should refer to the original published version of the material. 